\title{
Interactive comment on "Magma ascent mechanisms in the transition regime from solitary porosity waves to diapirism" by Janik Dohmen and Harro Schmeling
}

\section{Anonymous Referee \#2}

Received and published: 9 September 2020

\# General comments

The submitted manuscript systematically investigates magma ascent dynamics in order to capture the transition from the solitary wave regime to diapirism. The authors explore this transition by varying the relative compaction length of the system - here by changing the model extend dimensions while keeping the compaction length constant. Investigating fluid transport mechanisms in Earth subsurface is of broad interest with applications not only limited to melt in the crust, and thus the study is a welcome contribution. Although the title and abstract sound promising, the study presents several important issues that need to be addressed before to be further considered for 
publication.

1. The study's design The authors claim to resolve the transition from solitary wave of porosity to Stokes-like diapiric rise of magma. These two regimes are very different. The solitary waves of porosity occur in two-phase medium, when the fluid has a relative velocity compared to the solid. The diapiric ascent occurs if the fluid has no or very limited mobility with respect to the solid and thus the medium behaves as single-phase. The authors report here briefly the two-phase flow equations they rely on, which permit to resolve the two-phase motion. However, it is unclear what happens in the single-phase flow limit. In this limit, the equations should reduce to the single phase (Navier-) Stokes system. This part is totally absent from the study, both in the physical description (system of equations) and from the numerical implementation. The authors overlooked a study from Scott (1988) investigating a very similar research question, namely "The competition between percolation and circulation in a deformable porous medium". This short communication may be highly relevant and may support or challenge some statement claimed by the authors.

2. The numerical implementation In this study, the authors rely on numerical modelling to investigates the effect of changes in compaction length, or rather vary the domain size keeping the compaction length fixed. Being a numerical study, the current manuscript seriously lacks in robust model description, numerical implementation, benchmarking. These (non-exhaustive) steps are the basic technicalities one is expected to report when performing numerical experiments. The authors emphasise both in the Abstract and the Introduction the numerical challenges relative to accurately resolving fluid migration in the subsurface. However, no further discussion about numerical method, implementation, benchmarking, sensitivity analysis, etc... is present in the manuscript. The model configuration is poorly described and some basic information such as the numerical grid resolution should be reported in a well-crafted "Numerical Implementation" section well before the final discussion. Although focus should not be on benchmarking, ensuring accuracy of the numerical scheme and re-

Interactive comment
Printer-friendly version

Discussion paper 
lated results is primordial in studies like this one. As reported recently by Räss et al. (2019), lack of numerical resolution may lead to erroneous results. I am afraid that part of the results reported in this study are under-resolved, as at least a few tens of gridpoints are needed per compaction length to obtain accurate results. Also missing is the description of the transition from two-phase flow to single-phase flow. How do the authors treat the very small compaction length limit? In this limit, Stokes flow is dominating, and the motion of the fluid pocket needs advection of the solid matrix. There is no information regarding this important point in the manuscript. The governing equations are very cryptic, and it would be very helpful to see the finally implemented closed system of equations that is actually solved numerically, together with information on the numerical scheme that is used.

3. The quality of the reported results The reported results are interesting but in light of the previous comments, further work would be welcome to refine the Results and Discussion sections. The authors could put some additional efforts in crafting better quality figures. There are missing labels, fonts are very small and hardly readable in some cases, and figure captions display repetitions and miss important details. Also, it may be interesting to report in form of quiver plots the solid and fluid velocity components as those could be directly compared to results obtained by Scott in 1988.

To summarise, this manuscript tackles an interesting and not yet fully resolved question, but the study's design, numerical implementation and overall quality should be seriously improved before being considered for publication. Addressing these issues are important as in the current status it is hard for the reader to discriminate between resolved dynamics or numerical artefacts, especially in the transition regime. In the Discussion, the authors provide some insights in the challenges related to resolving the two-phase dynamics for large domains (or small compaction length). There may be a conceptual study design issue there. The authors spell out all the pitfall and they don't, but their study actually reports results that exactly suffer from those drawbacks. and may not be accurate. A potential way to improve the study would be to move a

Interactive comment
Printer-friendly version

Discussion paper 
large part of the issues raised in the discussion to the Section 2. For example, the discussion about the numerical grid resolution should appear much earlier. Then, one could discuss the issue, try to solve it. And if results cannot be trusted, then one should identify them and discard them from the analysis.

\section{\# Detailed comments}

I.18: In the current status, these may be numerical artefacts as well. Appropriate benchmarking would be welcome (e.g. running a test setup at various resolutions and reporting the results).

I.21: For accurate results of porosity waves, numerical resolution should always be such to have about 10 grid points per compaction length.

I.23-24: True, one should be careful. Please report how you carefully addressed these resolution issues.

I.47-49: Important question on "what are the numerical implications on modelling magma transport". Within the manuscript, however, these implications are discussed but it appears that the suggestions provided are not followed by the authors themselves.

Section Introduction: Please update it putting your contribution in light of previous work such as Scott (1988) and other potential studies.

eq.8-11: These are non-intuitive formulation of the momentum balance. What do v_1 and v_2 stand for? Please take some place to better describe the approach.

Section 2.2: Please complete the model setup.

I.85: What value of $A$ do you use in the experiments?

I.90: This may be problematic as number of grid points per compaction length will decrease with increased nondimensional box size. 
I.92: Can you precise what out and inflow conditions you use for the solid? Majority two-phase flow simulation apply free slip boundary conditions for the solid or porous matrix. Please clarify the model configuration - this is crucial for reproducible science.

I.93: What do mirroring boundary conditions refer to?

1.98 + eq.14: Please provide relevant reference for the Stokes velocity?

Interactive

comment

1.99: Please justify the choice of the radius you utilise in the Stokes formula.

eq.15: Ar not defined

Section 2: Besides the model setup, please report what final equations are implemented in the numerical model. Please also report about your numerical implementation, discretisation, solution strategy; all standard components one is expected to see in a numerical study that would enable reproducible science.

I.117: This may indeed show lack of numerical resolution.

I.121-126: No focussing is expected for linear shear and bulk rheology. The focussing you report here may rather be attributed to the still transient state of the model evolution - maybe due to the coarse resolution. To verify this, a higher resolution simulation on a larger domain should be carried out and running until the shape stabilises.

I.128-132: Why to report various analytical values when your simulation was carried out only with $n=3, m=1$. This only confuses the reader.

I.164.167: Internal circulation would be great to see in a figure. It is difficult to assess and acknowledge your findings based on text only.

I.170-171: How can you neglect the density difference between solid and melt. This should be the driving force.

Printer-friendly version

I.218: This conclusion should be verified by a higher resolution run.

Discussion paper

Section 3: May need further development upon updated results 
I.224-248: Interesting insight but all these hypotheses should be tested within appropriate modelling framework including spatial variations in the suggested material parameter fields and using sufficient numerical grid resolution to allow resolving the smallest features. Also, note that focussing will only occur if there is asymmetry among compaction and decompaction of the porous matrix, i.e. for non-linear rheology.

I.249-264: Good point, but it seems that this study exactly shows the reported artefacts in the results.

\section{\# References}

Scott, D. R. (1988). The competition between percolation and circulation in a deformable porous medium. Journal of Geophysical Research: Solid Earth, 93(B6), 6451-6462.

Räss, L., Duretz, T., \& Podladchikov, Y. Y. (2019). Resolving hydromechanical coupling in two and three dimensions: spontaneous channelling of porous fluids owing to decompaction weakening. Geophysical Journal International, 218(3), 1591-1616.

Interactive comment on Solid Earth Discuss., https://doi.org/10.5194/se-2020-124, 2020. 